\title{
Relier les études et la vie
}

Introduction

\section{Laurence Cornu}

\section{(2) OpenEdition Journals}

Édition électronique

URL : https://journals.openedition.org/ries/2060

DOI : 10.4000/ries.2060

ISSN : 2261-4265

Éditeur

France Education international

Édition imprimée

Date de publication : 1 septembre 2011

Pagination : $39-50$

ISBN : 978-2-85420-592-3

ISSN : $1254-4590$

\section{Référence électronique}

Laurence Cornu, "Relier les études et la vie », Revue internationale d'éducation de Sèvres [En ligne], 57 | septembre 2011, mis en ligne le 01 septembre 2011, consulté le 05 juillet 2021. URL : http:// journals.openedition.org/ries/2060 ; DOI : https://doi.org/10.4000/ries.2060 


\section{Le plaisir et l'ennui à l'école}

\section{Introduction}

\section{Relier les études et la vie}

\section{Laurence Cornu}

Que les élèves réussissent à l'école, et que l'école réussisse à les faire réussir au delà même de ses murs : voilà à la fois des aspirations individuelles et des visées de bien commun légitimes et non incompatibles. On cherche aujourd'hui à mesurer cette réussite par l'évaluation des performances des élèves et des établissements. Si le principe et les modalités de telles évaluations sont un sujet en soi (et un objet controversé, la définition des critères de réussite appelant aussi la réflexion), le comité de rédaction de la Revue internationale d'éducation de Sèvres a souhaité aborder (ou peut-être "border») cette question de la réussite à partir de la thématique du plaisir et de l'ennui à l'école : chacun peut en effet imaginer que réussir à l'école n'est pas étranger au plaisir que l'on a de venir dans le lieu scolaire, ni à celui d'apprendre, et cette considération commence à entrer dans les études sur l'efficacité des systèmes scolaires. L'on sait aussi que les « décrochages ", dont on se préoccupe avec raison aujourd'hui, traduisent une perte de sens de l'école, et même peur, dégoût, ennui, au sens fort du terme. L'intérêt d'une telle thématique est en fait de se demander ce qu'on attend de l'école, en différents lieux du monde, ce qu'on en attend au jour le jour, et pour "plus tard», dans son expérience quotidienne, et dans une projection temporelle. Le pari de ce dossier est de le faire non pas tant en essayant "d'objectiver" la dimension de motivation ou de répulsion de ces " affects », mais en donnant la parole aux acteurs mêmes, en pariant sur l'intérêt, et même l'importance, d'une évaluation essentiellement subjective, autrement dit d'une appréciation personnelle, non seulement comme objet d'étude mais comme expression d'un sentiment et d'un jugement de réalité, comme point de vue.

\section{L'EXPÉRIENCE DE SUJETS SENSIBLES}

Bien que la question pédagogique soit centrale et la controverse classique, l'écueil à éviter était de retomber dans l'ornière d'une énième polémique dichotomique sur le thème. Le choix était plutôt de suspendre toute 
précipitation de jugement moralisateur, et de s'enquérir simplement, factuellement, des impressions et conditions d'une fréquentation heureuse ou pénible de l'école, précisément à un moment où se répandent des études sur la souffrance des élèves et des enseignants, et des préoccupations de "bien-être " à l'école. Et le choix était surtout de chercher ce que les « sujets » peuvent en dire, les sujets collectifs, si l'on peut dire (les sociétés dans leurs débats, les pays dans leurs politiques institutionnelles), et les sujets individuels : enseignants et responsables, mais aussi, sinon d'abord, élèves. D'où ces termes de la langue courante, "plaisir et ennui ", qui suggèrent une attention portée à la qualité d'une expérience.

Remarquons et vérifions ceci : ce thème évoque pour chacun, immédiatement, sa propre expérience d'écolier et d'élève, et il suscite l'expression d'une appréciation de l'école, sur le moment, et pour ses conséquences supposées. L'essai de ce "sujet » sur un interlocuteur le plonge aussitôt dans un afflux de souvenirs, souriants ou amers, atmosphériques et précis à la fois, uniformes ou divers - toujours vifs après les années : la dimension sensible de l'expérience scolaire, garde, bien des années après le moment où elle polarisait tout le vécu de l'écolier, une force étonnante, et il arrive même que son évocation rétrospective plonge l'ancien élève dans le bilan de l'aventure intellectuelle, du « contentement » existentiel, ou du sentiment durable d'échec qu'il en a eu, touchant son être bien au delà d'effets concrets. Le " rapport » sensible de chacun de nous à l'école se révèle avoir été décisif, au point de l'avoir engagé dans la poursuite ou l'abandon de ses études - le terme d' " engagement » apparaît d'ailleurs dans plusieurs articles de ce dossier. Chacun sait en outre immédiatement (même si confusément) que ce n'est pas une alternative (le plaisir ou l'ennui), mais un étrange nouage qui se joue, entre plaisir et ennui, entre de multiples formes de plaisir et certaines formes d'ennui : le texte inaugural de Mona Ozouf nous en ouvre l'épaisseur d'expérience, en énonce les profondeurs d'enjeux et en distingue les subtilités de nuances, en même temps qu'il confirme que ce thème offre une entrée inattendue dans l'école comme phénomène historique, mondialisé, objet de fortes attentes contemporaines.

\section{UNE ENTRÉE SUBJECTIVE POUR EXPLORER « L'OBJET ÉCOLE ॥}

Il s'avère ainsi plein d'enseignements d'emprunter (une fois est si peu coutume), la " subjectivité » pour explorer ce dispositif universel de subjectivation - de formation des sujets - qu'est l'école. L'expérience des sujets sensibles que sont l'écolier, l'étudiant, le professeur, nous apprennent en effet quelque chose de l'objet école comme espace habitable, vécu, investi d'espoirs ou de désespoir, quelque chose qui traduit aussi des traits de son histoire, de sa dimension de pari sociétal et de choix politique. Plaisir et ennui, comme marqueurs sensibles d'une confiance ambivalente dans l'école aujourd'hui, comme témoins 
d'une crédibilité paradoxale de ce dispositif mondialisé de formation des jeunes générations?

On a proposé aux contributeurs un questionnement général : sur quelles lectures de l'école dans le monde pouvait ouvrir cette question du plaisir et de l'ennui qu'on y éprouve ? Un ou plusieurs de trois " observatoires » ont été suggérés : celui des discours collectifs ou communs (textes institutionnels, débat public, politiques de l'école), celui du témoignage des acteurs, celui des pratiques dans les lieux de la vie scolaire, (classe ou établissement). Les notions connexes (non imposées) étaient nombreuses, et le champ potentiel de réflexion fait de strates distinctes et de lopins variés, comme en témoigne la précieuse étude bibliographique de Bernadette Plumelle. L'ampleur du thème pouvait s'échapper dans l'enquête infinie. Or la question posée à travers le monde, dans l'incomplétude assumée du recueil, nous a en fait valu une réjouissante diversité de réponses, et d'étonnantes convergences.

On peut naturellement lire les textes dans l'ordre que l'on veut. Le dossier a été composé comme suit, à partir même de la diversité inattendue de la moisson : deux articles sur plaisir et ennui (France, pays de l'OCDE), puis trois articles d'approche synthétique sur des pays (Inde, Sénégal, Norvège) ; deux articles davantage centrés sur la classe et la vie d'un établissement, (comparaison Angleterre-France, États-Unis) ; trois articles enfin sur l'expérience des acteurs (étudiants au Brésil, professeurs au Portugal, un professeur en France).

\section{Plaisirs DIVERS, FORMES D'ENNUI ET ANXIÉTÉ}

On ne peut imaginer plus fort contraste entre les deux premiers textes. L'un attentif à l'expérience concrète, singulier et universel à la fois, tissant mémoire et histoire, dans une tonalité philosophique, mêlant ouverture, émerveillement et nostalgie. L'autre aussi quantitatif que le premier est " qualitatif », faisant état de statistiques objectivant des performances, des temps de lecture et des taux de réussite, dans un langage hétérogène au style du premier. Nous avons voulu ce contraste, témoignant de deux conceptions, sinon deux pratiques d'écriture, car cette hétérogénéité fait justement ressortir leur rencontre sur un thème : celui de l'importance du plaisir de lire, thème connu, et cependant ici revisité dans une nouvelle donne.

Le texte que Mona Ozouf nous a fait l'honneur de nous confier nous fait imaginer et remémorer mille plaisirs joueurs et aussi émerveillés de la petite école, pour les enfants démunis qui y découvraient le monde, en même temps qu'il évoque, avec Julien Gracq, la " géhenne » des collèges. De l'ennui « mortel » des journées d'école vécu par certains, il détache un "bon ennui », qui naît dans les intermittences de l'école : temps vide propice à la rêverie, à la " pensivité ", et à la lecture, temps dont le vide semble faire peur aux parents et aux enseignants d'aujourd'hui, qui le saturent d'occupations. C'est ce temps libre qui engageait à lire. 
Et tout indique, dans les études de réussite scolaires analysées par Sophie Vayssettes et Éric Charbonnier, que le plaisir de lire est déterminant pour «l'engagement » dans les études : il s'auto-alimente, se renforce en se développant, il permet une spirale positive, au point "d'atténuer l'effet d'un milieu familial peu aisé et peu instruit ", pour les élèves aimant lire, et lisant par plaisir : l'étude des données de l'enquête PISA 2009 le montre, d'où la suggestion de voir là un levier politique important. Resterait à trouver les « déclencheurs » du plaisir de lire, et d'abord de l'occasion de lire. Un temps ni quadrillé, ni sur-occupé, d'un côté, mais aussi, suggèrent ces deux auteurs, un environnement scolaire incitatif. Comme si, quand la lecture en solitude est en péril, il y avait lieu non pas tant de l'opposer à l'activité, mais d'y faire venir l'élève pour les besoins ou avec les plaisirs de l'activité, et dans une sociabilité inventive (organiser des clubs de lecture, etc.).

Le raisonnement se synthétise ainsi : réussir suppose de lire, et lire par plaisir accroît la réussite (tandis que ceux qui lisent sans plaisir peinent davantage). Or l'enthousiasme pour la lecture diminue. Il faut donc encourager non seulement la lecture, mais le fait de lire par plaisir, hors la classe. Mais comment ? Presque par tous les moyens du bord, toutes les ingéniosités pédagogiques qui puissent " accrocher " les candidats au décrochage.

Mais pourquoi donc lire rebute-t-il celui même qui, une fois quelque expérience faite, pourra y trouver grand plaisir ? Il y a, massive et préoccupante, la difficulté engendrée par l'impact contemporain de l'image et la fascination de l'immédiateté, qui rend l'attention des enfants et des adolescents si difficile à conquérir, comme le rappelle Mona Ozouf. Mais si, de l'extérieur, l'occupation consommatoire des cerveaux ne facilite pas (!) l'accession aux plaisirs de l'esprit, si la frénésie de réactivité peut en tuer les patiences, il se passe, en outre, que de l'intérieur rien n'est gagné : ce qui gâche le plaisir d'apprendre, dans son principe universel et ses multiples formes individuelles et culturelles, est ce que génère une forme d'organisation du système scolaire. Se précise par là même que l'opposé du plaisir n'est pas l'ennui mais la douleur ou, dans le registre psychique, la souffrance. Un des obstacles au plaisir d'apprendre et d'aller à l'école est interne à l'école, et réside dans l'anxiété que sa fréquentation génère. Ainsi, en France, c'est « l'anxiété d'avoir de mauvais résultats qui fait perdre une partie du bénéfice engendré » (par la dynamique de la lecture-plaisir). C'est ainsi que le thème de l'anxiété est un fil rouge - ou noir - de ce dossier.

Qu'en est-il hors OCDE ? Les trois textes qui suivent nous invitent au voyage dans les écoles de l'Inde, du Sénégal, de la Norvège.

\section{ÉCOLE, DESTINS D'ENFANTS ET CHOIX DE SOCIÉTÉ}

L'Inde : 180 millions d'enfants scolarisés à l'école primaire, mais aussi 8,15 millions d'enfants non scolarisés «pour qui la question du plaisir et de 
l'ennui ne se pose même pas ", sans compter le problème de la faible scolarisation des filles... Ce n'est pas sans quelque pointe amusée que les auteurs, D.S. Muley et Daya Pant, évoquent une France "fin prête à remplacer le PNB par l'ennui public comme instrument de mesure du bonheur de la population... », ni sans réalisme qu'ils mentionnent des enfants pour lesquels le motif d'aller à l'école est de pouvoir manger, d'autres encore qui se sont résignés à son ennui, et sortent le soir des écoles en retrouvant leur joie de vivre. Ces données constituent le fond sur lequel se détache une analyse à la fois prudente et aiguë. La mention des programmes d'extension générale de la scolarisation est l'occasion de signaler deux points décisifs : si l'apprentissage formel qui caractérise l'école "se fait dans la douleur », comparé à l'apprentissage informel ancestral, un mot d'encouragement de la part des maîtres peut faire toute la différence : "L'enseignant joue un rôle central dans l'apprentissage à l'école ». Or selon une étude, les enseignants passeraient moins de $2 \%$ de leur temps à féliciter leurs élèves, qui trop souvent les craignent et ne peuvent leur faire confiance pour les protéger de brutalités. D'autre part, témoignages et études incriminent des programmes surchargés, des apprentissages sans sollicitation de la réflexion de l'élève, et l'effet anxiogène d'examens permanents. Ainsi les politiques entreprennent-ils de construire ou d'améliorer les infrastructures, mais c'est tout autant sur les formes de maltraitance à l'école qu'il y a lieu d'agir, et sur la formation des enseignants. Voici enfin ce que permet de découvrir cet article : le point de vue des élèves, si peu entendu, révèle avec justesse les ombres d'un système, qui n'est pas particulier à l'Inde : « J'aime bien la chimie, mais ma prof est très sévère. Elle s'énerve quand je pose une question. C'est très frustrant. Je préfèrerais ne pas faire de chimie ».

Présentant son pays, le philosophe Abdoulaye Elimane Kane nous en donne une vue diachronique, resituant le problème dans l'histoire de l'école au Sénégal : imposée par le colonialisme, "l'école où je pousse mes enfants tuera en eux ce qu'aujourd'hui nous aimons et conservons à juste titre ", dit un personnage de roman. Voici donc un autre thème de ce dossier, celui de l'opposition entre apprentissage informel et formel, entre une forme de vie et une autre, entre une école qui garde de la tradition grecque un mode de transmission théorique lié au statut second de la pratique, et une culture qui sait les unir. Déphasage complet pour un jeune berger dont «le métier ne manque certainement pas de contraintes et d'ennui ", mais qui alors décroche du temps engrillagé de l'école. Mais l'école ne garde pas seulement la trace de cette violence initiale du contact entre deux cultures. D'une part elle est aujourd'hui indispensable pour l'essor du pays, et les autorités du pays travaillent à l'extension de la scolarité, et d'autre part son succès dépend d'une valorisation du savoir comme idéal, qui est prégnante au Sénégal, sous de multiples figures, qui ne sont pas seulement occidentales, et qui émergent aussi du pays même : cette valeur fut portée par l'influence musulmane au XIX ${ }^{e}$ siècle. Enfin elle a été illustrée de 
manière originale et magnifique dans son histoire politique par des hommes tels que Léopold Sedar Senghor. Le Sénégal aurait en quelque sorte l'opportunité de réunir ses traditions historiques de connaissance et de valorisation sociale du savoir. La question est alors de remettre en relation ce que l'apprentissage formel a séparé pour déployer sa puissance. «Relier l'étude et la vie » : ce texte aborde ainsi encore dans une réflexion subtile et profonde l'inventivité pédagogique indispensable à sa haute idée de l'école. Pas d'opposition ici, ni de dichotomie, ni d'alternative, entre l'idée philosophique et politique de l'école et l'invention pédagogique que sa mise en œuvre vivante appelle.

On découvre ainsi avec l'auteur combien plaisir et ennui « qui sont certes affaire d'individus et de circonstances " impliquent une "dimension historique et culturelle ", et que " décider collectivement et/ou individuellement de la fréquenter se pose fondamentalement en termes de volonté et de choix d'un mode d'organisation de la société et d'insertion dans la vie sociale. »

Décision collective, enjeu de société autant que de destin individuel, cette dimension de choix sociétal, qui engage un pays, c'est aussi, dans un contexte bien différent, ce que montre l'article d'Anne-Lise Høstmark Tarrou consacré à la Norvège. Voilà un pays où le fait de "se sentir bien à l'école » a été explicitement identifié comme un facteur de réussite, et officiellement encouragé. Plaisir ? "Bien-être à l'école » : il n’y a rien là de consumériste, mais la simple prise en compte de la qualité d'un cadre, où l'on fait vivre les enfants de longues années, et où il est décisif pour un enfant d'être " accepté par les autres élèves, et mis en valeur par les enseignants ». Il y a donc eu depuis une trentaine d'années des efforts institutionnels sur ce thème, associés à une lutte visant à neutraliser les inégalités scolaires dans le quotidien même de l'école. D'où le choix de ne pas noter jusqu'à un certain niveau de classe. Or la situation de la Norvège ici analysée met en évidence des paradoxes ou des difficultés rémanentes. Les décrochages ne sont pas absents de l'école norvégienne, créant à nouveau un débat sur la notation et sur la réussite des élèves. Là encore le témoignage directement recueilli des élèves éclaire un pan du problème: l'apprentissage est bien mieux accepté et assimilé lorsqu'on « comprend de quoi il s'agit». Une approche pratique des contenus enseignés semble le permettre. Pour autant, " il ne semble pas opportun de réduire l'apprentissage des matières générales mais il y aurait lieu de les mettre en perspective autrement "... C'est la raison d'être des connaissances qui manque à certains, non pas l'énoncé d'un discours à vrai dire, mais la saisie d'une "raison sensible »: une fois ce lien établi, le plaisir de faire des maths est là de nouveau. Ce lien ne signifie pas seulement, ou pas exactement, une approche utilitariste, mais la mise en rapport de la connaissance avec une situation sinon vécue, du moins imaginable, désirée peut-être, en tout cas pourvue de signification pour l'élève : question non pas tant d'instrumentalisation que de sens, de greffe sur le sensible de l'expérience et le désir de se réaliser, de raison d'être du détour, de motif pour l'effort 
demandé. Ne serait-ce pas là aussi relier l'étude et la vie ? Mais de quelle vie parle-t-on? Les savoirs ne se suffisent-ils pas à eux mêmes?

\section{ACTIVITÉ, SOCIABILITÉ, SOLITUDE ? MODÈLES ANGLO-SAXONS ET FRANÇAIS}

On rencontre inévitablement, dans une telle thématique, la controverse qui fait s'affronter tenants des vertus de l'ennui et tenants d'une pédagogie ludique, ainsi que la discussion parallèle qui oppose les modèles anglo-saxons et le modèle «continental ». La controverse est ancienne qui opposerait d'un côté Montaigne, Rousseau, et de l'autre Hegel dans les Principes de la philosophie $d u$ droit (\$175), ou encore Alain, dans les Propos sur l'éducation, ces deux derniers également critiques à l'égard de la puérilité d'approches "ludiques ", qui n'honoreraient pas le "désir de grandir " des enfants eux-mêmes. À vrai dire, ces pensées sont plus subtiles que la caricature de leur opposition, et ici, la description comparative que Maroussia Ravaud fait des classes française et anglaise permet d'apporter quelques nuances dans le contraste visible de l'organisation des espaces de classes, également repérable dans les discours pédagogiques. En Angleterre, l'approche pédagogique holiste qui «fait du bonheur de l'enfant un objectif en soi et considère le bien être comme un pré-requis de l'apprentissage » a été " accueillie avec enthousiasme par une commission parlementaire influente ». "L'enfant au centre des apprentissage », voilà qui a moins " pris » en France que la " child centred education », dont la perspective ne pose aucun problème outre-Manche ou outre-Atlantique. On peut être tenté par une interprétation "culturaliste ", toutefois les "traditions " évoluent : le terme de plaisir n'est pas absent des textes français qui pourtant insistent sur le " travail ». La recherche du plaisir d'apprendre ou de conditions agréables pour apprendre n'est pas nécessairement la célébration d'une pédagogie superficiellement ludique. Dans les deux cas, "c'est comme moyen qu'est mobilisé le plaisir », et comme facteur supposé de réussite. Critère pragmatique alors, ("cherchons ce qui "marche" ») ? En réalité, les effets bénéfiques ne sont pas nettement hiérarchisés: alors que l'école française «cultive une rhétorique de l'effort », la rhétorique du plaisir rencontre comme limite une réalité de l'ennui ou une nécessité de l'effort que l'école anglaise a du mal à justifier. Enfin apparaît ici une contradiction interne aux évolutions du système école dans la mondialisation comparative des évaluations: l'évaluation des établissements va-t-elle améliorer la qualité de l'accueil des élèves, (comme les programmes indiens le font valoir) ou va-t-elle augmenter l'âpreté des concurrences et le stress des résultats?

L'entretien de Pierre-Louis Gauthier avec Karali Pia Kitzele, proviseur d'une High School à Brooklyn, permet de découvrir une politique d'établissement qui se rattache explicitement à une "culture américaine des loisirs et du jeu ». 
On sait les mots très durs de Hannah Arendt sur les méfaits, selon elle, d'une pédagogie par le "faire», et cantonnée au monde de l'enfant. Mme Kitzele justifie l'importance (étonnante aux yeux d'un Français) d'une vie d'établissement appuyée sur les hobbies des adolescents, et qui vise à " renforcer la communauté scolaire ", à " écarter l'ennui à tout prix ». Développer l'attractivité des distractions - le divertissement pascalien -, voilà qui risque d'écarter non seulement l'ennui mais le "bon ennui », fertile à la pensée. Ainsi formulé, ce projet volontariste d'établissement apparaît être aux antipodes de l'école de Mona Ozouf. On pourrait forcer le trait: nouveau monde contre Europe, nouvelles générations contre nostalgie d'une école qui n'existe plus, communauté versus formation individuelle, particularismes versus universalisme, activité permanente versus penser par soi-même... Sans doute y a-t-il là quelque chose qui paraît étrange - et pour d'autres, attirant - au "vieux continent ». La comparaison entre les deux systèmes aboutirait-elle, après fascination, comme pour la justice américaine, à la conscience de l'incompatibilité des logiques ? Si l'on veut ne céder ni aux dépits d'un échec, ni aux illusions d'une harmonisation, ni aux mirages d'une colline plus verte ailleurs, il vaut la peine de se souvenir que le collège était mentionné comme un lieu de souffrance par Mona Ozouf, et que le collège est le maillon en difficulté dans beaucoup de système scolaires. Il y a ici une attention portée au lien collectif des élèves, à un moment où, adolescents, ils ont besoin de cette dimension pour continuer l'effort que la fin de la phase de latence exige d'eux. Il y a dans le collège de Brooklyn un « effort » pour " relier l'enseignement au monde réel des élèves, et le rendre pertinent par rapport à leur vie ». L'opposé de l'ennui est ici un « au delà du plaisir », appelé « engagement » : s'engager dans ses propres études. Cette décision est personnelle, solitaire. Mais est-elle isolée? L'étayage d'une sociabilité est ici recherché, qui a ses formes «culturelles », et ses variantes pédagogiques. Reste à préciser encore de quels plaisirs on parle, de quelle vie aussi : "relier l'étude et la vie », est-ce la même chose que de « relier les études au monde réel des élèves »?

C'est en écoutant les acteurs que l'on peut s'en faire une idée : trois articles enfin recueillent ou expriment, dans des approches différentes, leurs points de vue.

\section{PSYChANALYSE DE L'INSTITUTION, SOCIOLOGIE DE LA SOUFFRANCE ET LITTÉRATURE}

Du Brésil nous parvient une enquête faite par Vera Esther Ireland, psychanalyste, sur le plaisir et la souffrance des étudiants. Le rappel de la théorie psychanalytique fournit un cadre d'analyse des affects, mais aussi de la situation : les étudiants seraient "métaphoriquement en présence d'une institution mère (l'école, l'université) ». Les propos des étudiants sont rapportés de façon factuelle ou synthétisés dans leur teneur manifeste. L’enjeu est triple: (1) mettre en 
évidence la clarté de perception des difficultés rencontrées : raisons de la souffrance, formes de la satisfaction, complexité ou ambivalence des sentiments, jugement sur l'importance des enseignants etc. ; les étudiants sollicités expriment des remarques de bon sens, simples, qui sonnent juste ; (2) faire une lecture anthropologique de la situation : montrer que l'engagement des étudiants dans leurs études garde la trace ou la structure de l'expérience de satisfaction/frustration du bébé (faire avec la frustration, s'accommoder de l'objet, trouver des objets transitionnels) ; la psychanalyse permet de lire ainsi la problématique de l'éducation et des objets de culture : si l'expérience de la frustration est inévitable pour le sujet humain, elle n'est psychiquement instituante qu'à certaines conditions de la part de l'entourage ; 3 ) proposer que les institutions, comme une " mère suffisamment bonne ", se mettent à l'écoute de ceux dont elles ont la charge : ce n'est pas ici l'apologie d'un maternage ni d'une indulgence maternelle que d'autres opposeraient à la rigueur des figures de la loi (du père). Avec de la loi symbolique, il doit pouvoir y avoir de l'écoute, et une écoute de la souffrance.

Les enseignants enfin souffrent, et si les sociologues s'y intéressent, comme le font ici José Alberto Correia et João Caramelo, c'est d'une part que les auteurs y voient des effets structurels de la crise de l'école, et d'autre part qu'ils en proposent une typologie des manières d'y répondre : question de récits et de grammaires. Les "récits " dominants de la crise de l'école voient paradoxalement, comme solution, davantage d'école. Mais dans la montée de "l'évaluocratie ", les professeurs se retrouvent étrangers à leurs élèves et au sens qu'ils peuvent donner à leurs efforts : les auteurs voient un problème " cognitif » dans cette perte d'un régime de familiarité. Ennui ? Plus profondément, souffrance dont les professeurs tentent de se protéger dans des idéologies "défensives ", et dont les auteurs décrivent trois formes, inspirées du triangle de Houssaye et marquées par une résignation dont tout plaisir semble absent. Ce qui est ici analysé au Portugal a des causes si générales, liées aux transformations de l'école dans la mondialisation capitaliste, que l'on ne peut qu'en imaginer l'extension ou en reconnaître déjà la justesse en d'autres lieux.

Des étudiants suffisamment heureux d'apprendre ont besoin de professeurs suffisamment heureux d'enseigner : le système fait-il encore confiance à ces derniers, suffisamment pour qu'ils fassent confiance à leurs élèves ?

L'analyse, par Anne Maurel, de l'ennui comme effet d'un ensemble d'attentes sociales, de clichés, d'immobilismes d'un système où tout est devenu "lettre morte " corrobore l'idée que le "plus d'école " que l'on prône semble s'être coupé d'élans de sens que soutenaient les utopies scolaires, et surtout de la raison vivante d'aimer lire, faire des mathématiques, dessiner, regarder, comprendre... Dans le "déclin de l'institution ", ce sont bien les sujets qui sont instituants, en traversant leur souffrance même et offrant à celle des élèves l'expérience de formes qui les expriment et les subliment. Contre la répétition mortifère, le professeur de lettres ici œuvre à rendre les textes à nouveau vivants, 
c'est-à-dire sensibles, parlants pour l'œil et pour l'oreille, non séparés du cœur et de l'esprit. La traversée de l'angoisse et les retrouvailles de l'émerveillement d'une rencontre esthétique et existentielle, il les éprouve lui-même. C'est sa voix qui redonne corps aux textes et relie les textes et la vie. Plaisir ? Creuser ainsi l'expérience de la vie en rendant vifs les textes, c'est faire faire l'expérience selon laquelle a thing of beauty is a joy for ever. De même, un enseignant de mathématiques peut-il encore espérer faire faire l'expérience de la joie intellectuelle qu'il y a à faire des mathématiques, à partir d'un problème. Comprendre et résoudre un problème : découvrir la puissance de son propre esprit, voilà qui permet d'engager l'effort parfois austère de l'étude. L'expérience de ce que l'effort à consentir vaut la peine est décisif, non seulement pour la satisfaction de réussir, mais pour la joie de comprendre, de ressentir, de grandir.

\section{LE TEMPS DE LA SCHOLÈ}

Le processus d'éducation, l'humanisation, est une institution longue et exigeante pour le vivant parlant, qui implique le renoncement à certains plaisirs et la valorisation d'autres, qui sont fonction de choix de société. L'école en est une invention historique. La valorisation désintéressée du savoir qu'elle pouvait représenter n'était sans doute pas la seule des finalités qu'on lui a politiquement assignées dans son histoire. Mais la valorisation instrumentale du savoir la met aujourd'hui en paradoxe, et en difficulté les nouvelles manipulations des plaisirs.

Comment la scholè - le temps désœuvré, libre de tout affairement, le «bon ennui » indispensable à la vie de l'esprit - est-elle encore possible ? Un premier enseignement de ce dossier est que cette entrée par l'expérience subjective de l'école (celle des sujets, et celle de ce que leur "fait » l'école), révèle quelque chose de ce dispositif désormais mondialisé : quelque chose de la crise de son récit, une inflation des attentes parallèle à une perte de crédibilité. La question du plaisir et de l'ennui à l'école n'est pas seulement une question de pays saturés de consommation, de professeurs supposés incompétents, d'élèves mal disposés, de pédagogies inadaptées, ni de seul apprentissage de frustrations nécessaires à toute vie de l'esprit, mais elle met en évidence le paradoxe profond et général d'une époque où l'on veut, partout dans le monde, à la fois plus et moins de scolarisation, et la position étrange d'une école universellement investie et en danger d'être subjectivement désinvestie.

Dans cette conjoncture étrange, peut-être pourrait-on lire dans le bouquet de ces textes un même centre de gravité " pédagogique » : si les uns et les autres n'entendent pas "plaisir " en un même sens, ils entendent tous relier l'école et «la vie ». Tous n'entendent pas «la vie » en un même sens, et chaque pays en invente ses voies propres. Mais ne pourrait-on dire que relier l'étude et la vie, c'est renouer les liens entre expérience et abstraction, entre sensible et intelligible? 
Aux processus d'apprentissage, il faut un «cadre ». Il apparaît nécessaire que ce cadre soit non seulement délimité, mais aussi accueillant, au sens architectural, et symbolique, au sens où l'on en prendrait soin. Hospitalier, habitable, pour des apprentissages vivants, qui peuvent alors être exigeants. Alors quelque processus peut se produire, qui a une part énigmatique, et cependant élémentaire : il suffit de peu pour dégoûter un élève (chacun ne le sait-il pas ?), il relève du bon sens de chercher à réunir quelques conditions nécessaires (même si non assurées d'être suffisantes), pour que l'alchimie d'un goût pour la chimie puisse opérer.

Ce que les disciplines exigent de concentration et de médiation, de renoncements aux distractions, n'a jamais été simple, et est aujourd'hui rendu difficile par la fascination de l'immédiateté, l'impatience, la labilité contemporaine des attentions. Il faut une anthropologie comme celle de la psychanalyse pour nous aider à penser et à reconstruire le sens des objets transitionnels, à prendre en compte l'investissement de la voix et les plaisirs du regard, l'importance des phénomènes de transfert, le jeu des malentendus et l'énigme des désirs de sujets. Si la vie de l'esprit suppose le renoncement à l'immédiateté sensible, reste que bien des médiations de l'esprit sont sensibles, que la "sublimation " est encore un plaisir, et que les processus intellectuels sont liés à des processus psychiques. Dans l'inquiétude que peut susciter la difficulté des élèves d'aujourd'hui à lire, il semble qu'il y ait lieu d'insister sur l'investissement sensible des activités intellectuelles, mais aussi sur la nécessité, l'importance vitale de penser ce que l'on apprend.

Relier l'étude et la vie, c'est rencontrer le désir de sujets vivants. L'école instrumentalisée peut bien instrumentaliser le plaisir. À se couper du désir des sujets, de leur capacité - celle des élèves comme des professeurs - à éprouver des joies esthétiques ou intellectuelles, sa contrainte, accompagnée d'injonctions pressantes, quand elles ne sont pas paradoxales, ne peut qu'engendrer de la souffrance, du rejet, contre tout sens commun. Ce qu'aura apporté ce dossier, pour finir, c'est, au delà des analyses, un trésor de témoignages, et la prise de conscience que l'on prête trop rarement attention à ce que peut dire un élève. Dans le temps de l'école, ne pourrait-il y a voir de moment pour la parole?

Bouquet final :

«C'est beaucoup plus facile d'apprendre les maths quand on comprend de quoi il s'agit. »

«Les examens n'évaluent pas l'intelligence d'un élève mais sa capacité à mémoriser. »

« Mis à part l'intérêt ou le désintérêt que l'on peut ressentir à titre individuel pour une matière comme source d'ennui ou de plaisir, on constate en fait que dans certaines matières, l'école suscite l'ennui le plus total et génère une profonde angoisse même chez les élèves les plus intéressés. Par exemple les établissements scolaires continuent d'entraver la réflexion des élèves en leur disant de s'en tenir au programme...» 


\section{BIBLIOGRAPHIE}

ANZIEU Didier (1994) : Du moi-peau au moi pensant. Dunod.

ARENDT Hannah (1994) : "La crise de l'éducation », in La crise de la culture, Folio Essais.

CORNU Laurence (2005) : "Autorité, hospitalité ", in La crise de la culture scolaire, sous la direction de D. Kambouchner et F. Jacquet-Francillon, Presses Universitaires de France.

DUBET François (2002) : Le déclin de l'institution, Seuil.

OZOUF Mona (2009) : Composition française, Gallimard.

STIEGLER Bernard (2008): Prendre soin de la jeunesse et des générations, Flammarion. 